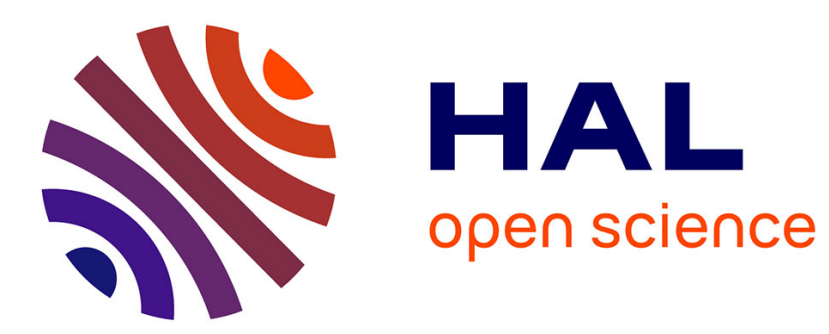

\title{
L'insertion du Centre Historique et de la zone urbaine du Canal dans l'aire métropolitaine de Panama
}

\author{
Anne Collin Delavaud
}

\section{To cite this version:}

Anne Collin Delavaud. L'insertion du Centre Historique et de la zone urbaine du Canal dans l'aire métropolitaine de Panama. L'Information géographique, 2006, 70, pp.21-35. halshs-00162654

\section{HAL Id: halshs-00162654 \\ https://shs.hal.science/halshs-00162654}

Submitted on 29 May 2012

HAL is a multi-disciplinary open access archive for the deposit and dissemination of scientific research documents, whether they are published or not. The documents may come from teaching and research institutions in France or abroad, or from public or private research centers.
L'archive ouverte pluridisciplinaire HAL, est destinée au dépôt et à la diffusion de documents scientifiques de niveau recherche, publiés ou non, émanant des établissements d'enseignement et de recherche français ou étrangers, des laboratoires publics ou privés. 


\author{
Anne Collin Delavaud \\ Professeur des Universités \\ Institut des hautes études de l'Amérique latine \\ Université de la Sorbonne Nouvelle- Paris III \\ a.collin@univ-paris3.fr
}

publié en 2006 dans L'information géographique $\mathrm{n}^{\circ} 70$

Dans le dossier : "Les nouvelle formes d'intervention dans les centres historiques en

Amérique latine"

\title{
L'insertion du centre historique et de la zone urbaine du canal dans l'aire métropolitaine de Panama
}

Avec près d'un million d'habitants, Panama ne semble pas encore ralentir sa croissance spatiale et doit, en même temps, assurer une forte recomposition urbaine du fait des bouleversements internes liés non seulement à la rétrocession de la zone américaine, mais aussi à la demande logistique et touristique internationale qui réclame, à la fois, de nouveaux quartiers fonctionnels et la réhabilitation de quartiers anciens. Ainsi, il ne s'agit pas ici seulement d'un processus classique de réhabilitation mais aussi d'une réinsertion globale du centre d'origine coloniale ainsi que celle de la zone urbaine américaine, deux quartiers fort différents mais patrimoniaux l'un et l'autre, dans une zone métropolitaine au service de la mondialisation.

En moins de 20 ans, la ville a récupéré la zone du canal restée sous la souveraineté américaine ${ }^{1}$ pendant près d'un siècle. L'organisation spatiale héritée de ce territoire, partiellement clos mais militairement gardé, a empêché l'extension de la ville panaméenne à l'Ouest sur une bande de $4 \mathrm{~km}$ et a modelé un espace urbain particulier aux activités logistiques et de défense. Dans ce contexte de deux villes voisines, l'une américaine, l'autre panaméenne, dotée chacune de leurs espaces centraux, la place pour le centre historique d'origine coloniale s'est trouvée mise à l'écart car ne répondant plus aux attentes de l'une ou l'autre. Qu'est devenu ce quartier avec la rétrocession et la dynamique métropolitaine récente? Comme dans les villes d'Amérique latine, des phases d'essor et de stagnation ont marqué ce territoire urbain emblématique mais victime de politiques urbaines en faveur du centre d'affaires et de son Centre Financier International, lui faisant courir des risques de disparition avant que ce patrimoine ne soit reconnu sur le plan national et international. Les grands projets du début de la décennie 2000 affichent une volonté de réintégrer ce quartier non seulement à l'espace urbain réunifié mais aussi d'en faire un des éléments moteurs du développement économique. Mettre en évidence les éléments de cette nouvelle réintégration permet de comprendre sur quelles initiatives tant de la part du secteur public que du secteur privé, ce projet urbain est tenté, au cours d'une période politique stable dans un contexte économique résolument libéral.

\section{Une évolution divergente pendant le XXe siècle}

Tout au long du siècle, le centre d'origine coloniale, limité encore fin XIX e siècle à San Felipe et à son "faubourg", s'est très vite agrandi de Santa Ana et d'El Chorillo, avec l'arrivée des travailleurs étrangers pour les chantiers de construction du chemin de fer (1850), du canal français (1884), puis du canal américain (1903). Trop petit et enserré de quartiers populaires, il connaît des phases de déplacement de ses activités et de sa fonction résidentielle vers la seule zone située à l'Est, au bord de la baie, puisque la zone du canal lui interdit une croissance vers 1 'Ouest dès 1903. Ce nouveau centre connaît les faveurs d'une exposition internationale dont les édifices construits deviendront le cœur d'une nouvelle ville administrative, barrio la Exposición, puis il avance à Calidonia (1930, et continue sa progression vers l'Est, à Bella Vista qui offrira des terrains pour les grands équipements des années 60 comme l'Hôtel Panamá et l'Université. C'est là, que commenceront à s'installer les banques qui formeront le Centre des Affaires Internationales (1970). Les familles aisées ont quitté les

\footnotetext{
${ }^{1}$ La zone du canal et de ce fait la frontière à la croissance de la ville de Panama a été délimitée en 1913
} 
immeubles anciens du centre colonial puis de Calidonia, pour des maisons confortables entourées de jardin dans les collines alentours et dans la presqu'île de Paitilla.

L'ex zone américaine qui abritait 50000 habitants environ, offre un paysage urbain de grande qualité marqué par la dispersion des petits immeubles de style traditionnels panamo-américains dans un vaste parc d'arbres tropicaux et de pelouses très bien entretenues sans aucune clôture. Territoires d'opérations pour la navigation sur le canal et pour les zones militaires, mais aussi espaces résidentiels et forêts protégées, ont pendant un siècle opéré comme une frontière matérielle, coupant non seulement le pays, mais aussi, la ville en deux territoires bien séparés par le canal et sa zone, franchissables seulement aux trois écluses en attendant la construction du Pont de la Liberté à l'entrée du canal sur le Pacifique qui facilita, à partir de 1970, le franchissement du canal et l'apparition de nouveaux lotissements urbains de l'autre côté.

Deux mondes cohabitaient sans espace de transition, deux modes d'habiter correspondant à deux types de revenus, à deux histoires parallèles. Les premières années 2000 changent les données définitivement et autorisent une recomposition urbaine sur la base d'une meilleure articulation de ces deux quartiers au reste de la ville : un espace urbain d'origine coloniale largement reconstruit jusqu'en 1930, fortement dégradé depuis, et un espace aménagé tout au long du siècle pour répondre à une fonction productive et la sécurité d'une souveraineté étrangère. La recomposition de ces deux quartiers se fait au profit de l'ensemble de la ville ${ }^{2}$.

Les efforts d intégration de deux espaces urbains

L'administration des biens de la zone du canal est devenue panaméenne à la suite du processus de rétrocession commencé en 1979, suite au Traité Carter-Torrijos de 1977, a connu des étapes successives $^{3}$. Le retour à la démocratie met en place une nouvelle institution, créée en 1993, l'Autoridad de la Región Interoceanica (A.R.I.) qui a pour mission d'inventorier, de gérer et de vendre des biens fonciers et immobiliers de la zone du canal. Outre une reconversion civile de la zone, l'A.R.I. a le monopole d'intervention pour évaluer ces biens et les mettre sur le marché en tenant compte du plan d'occupation du sol proposé en 1996. Il s'agissait, d'une part, de conserver les espaces fonctionnels destinés aux opérations de navigation et à son commandement et, d'autre part, de mettre sur le marché des terrains, des hangars, des maisons et des édifices collectifs occupés par les Américains, dont 12 bases militaires). Sur les $1400 \mathrm{~km} 2$ que représente la zone américaine, la zone urbaine du canal représentant 12560 ha accompagnés de 3626 hectares d'espaces vert urbains ${ }^{4}$.

Réalisée sur plusieurs années, la rétrocession a facilité l'accès aux immeubles américains à de nombreux particuliers et administrations. Sur les 1522 maisons et édifices inventoriés, 800 sont attribués à des panaméens, les autres à différentes entités gouvernementales nationales et internationales. Toutes les maisons d'Albrook, Curundú, Diablo Cardenas ont été achetées ; il reste quelques édifices à vendre à Clayton. Les prix actuels sont élevés car l'entretien voire la restauration de ces maisons souvent en bois, ont ralenti la demande. Le quartier d'Ancón est resté lui administratif.

\footnotetext{
${ }^{2}$ Comme les autres villes d'Amérique latine, Panama connaît dans les autres quartiers les mêmes difficultés avec ses différences sociales et architecturales.

${ }^{3}$ La première de 1978, avec la création de l'Autorité du Canal de Panama (A.C.P.), chargé d'organiser l'usage de toutes les terres (Plan Maestro de usos del suelo para la zona y la cuenca del canal-1979, non approuvé suite à la cessation de l'ACP 1980) et des édifices U.S., puis, en 1981, sous l'autorité du Ministère de l'Intérieur pour l'administration des biens rétrocédés et le Ministère du Logement pour les édifices et plus particulièrement, les Fuerzas de Defensa de Panama, créées en $1983^{3}$. Les zones rétrocédées et le canal deviennent propriété militaire de la Garde, unique corps armé du pays qui disparaîtra, en 1989, après l'intervention U. S contre Noriega.

4 Il s'agit de plus de mettre en place une plate-forme multi-modale pour répondre aux besoins de la navigation maritime, en privatisant les services offerts par la Commission du Canal (US).
} 
A proximité du centre historique de la ville de Panamá, cet espace urbain est aussi un lieu de production indispensable à l'économie nationale et internationale. Se tromper dans son intégration, c'est risquer une catastrophe économique. Le contrôle militaire, pendant les premières années, a certainement permis au modèle urbain américain de continuer sans rupture. La rigidité de la propriété publique de la terre jusqu'à la réalisation du cadastre de la zone américaine a maintenu en l'état ces quartiers dont le paysage urbain est resté intact en dehors des zones de grands aménagements (ports, parcs à containeurs, terminal terrestre, centre commercial et échangeur).

La faible densité de la ville américaine aurait pu faciliter des occupations de terrain, or aucune invasion n'a été enregistrée dans cette partie alors qu'ailleurs elle a permis l'accès au sol urbain à de nombreux migrants. Seule une frange importante de la forêt protégée en limite du district d'Arraiján à l'ouest du canal a été envahie à partir de 1985. Aujourd'hui, l'A.R.I. et l'Autorité du Canal de Panama (A.C.P.) surveillent de très près les territoires sous sa responsabilité.

Vingt ans après, il faut reconnaître que la zone urbaine américaine répond aux normes du développement d'une cité jardin bénéficiant d'une faible densité du bâti. Le danger n'est cependant pas écarté, les habitants d'Albrook se battent pour ne pas voir leur paysage complètement transformé par des lotissements prévus sur la piste d'aviation désormais civile. Le projet de cité administrative a été abandonné par manque d'intérêt à regrouper une administration dispersée dans les centres successifs de la capitale. Il reste la demande résidentielle qui perce dans des nouveaux lotissements entre Clayton et Albrook Tout ce secteur bénéficie d'une hauteur limitée à cause de la piste d'aviation. Que se passera t'il lorsque la piste sera construite avec des tours aux appartements avec vues imprenables à la fois sur le canal, le Pacifique et la forêt protégée ? Le deuxième pont en construction en 2003 entraîne déjà des coupes dans la forêt pour laisser passer l'autoroute mais aussi, très certainement, pour accueillir des lotissements. La compagnie constructrice est aussi immobilière.

La mise à l'écart du centre ancien (casco antiguo)

La ville de Panamá, limitée encore à la péninsule, connaît deux périodes de prospérité avec la construction du premier canal par les Français, puis une deuxième période avec l'arrivée des Américains. Les constructions héritées de ces phases de prospérité témoignent du dynamisme urbain. La taille des monuments dans un espace pourtant restreint ( 44 hectares), les édifices de 5 étages et les activités commerciales tout au long de l'Avenue Centrale, reflètent une société en plein essor et une croissance démographique, 60000 habitants en 1914 à la date d'inauguration du canal construit par des milliers de travailleurs venus de partout et pour lesquels des édifices de bois à plusieurs étages seront construits dans les quartiers voisins (El Marañon, Chorillo). Les influences architecturales européennes, caraïbes et américaines sont prépondérantes dans le paysage entre 1880 et 1920 . Les Américains s'installent à l'Ouest, les gens aisés partent vers l'Est, laissant de côté ce lieu historique sombré dans une décadence injuste.

Les investissements se détournent de cette zone dont on ne connaît pas l'avenir, il était plus efficace de changer l'usage dans la zone centre pour obtenir une plus grande densité. Les Américains avaient d'ailleurs rendu la presqu'île de Paitilla et l'aéroport voisin dès 1960. Ses terrains jouxtant le Centre Financier attirent les investissements immobiliers qui continuent leur marche vers l'Est occupant mangrove, carrière et décharge 5 .

Les crises qui suivirent le firent oublier. Dans la décennie 70, il y eut un premier effort, avec la création de la Dirección del Patrimonio qui élabora un premier Plan Maestro ${ }^{6}$. Un renouveau est

\footnotetext{
5 A l'extrême limite orientale, Costa del Este, un lotissement de luxe de 300 ha sur une ancienne décharge est un projet sur plus de 15 ans alors que Las Garzas est une immense carrière envahie de l'autre côté de l'aéroport.
}

${ }^{6}$ Proyecto de Restauración del Conjunto Monumental Histórico del Casco Antiguo OEA- Flores Marini (1972-1976) . 
enregistré au début de la décennie 90, avec quelques achats de maisons par des personnalités locales mais sans avoir eu l'effet d'entraînement sur l'ensemble car la demande en maison ancienne de caractère était satisfaite par l'offre de l'ex zone américaine. Un projet de revitalisation est proposé en 1995 à la suite du Plan de Acción Casco Antiguo.

Le territoire officiel du centre historique comprend la totalité du corregimiento de San Felipe, 19 îlots du celui de Santa Ana et 14 celui de Chorillo. Les autres îlots de ces corregimientos qui constituent pourtant la ville de la fin du XIXe siècle ont été rejetés ${ }^{7}$. L'ensemble reconnu abrite un peu plus de 11 000 habitants (recensement 2000) dont 6928 pour San Felipe qui comptait encore, en 1990, 10282 habitants soit une baisse de $33 \%{ }^{8}$. Près de la moitié des habitants sont nés dans la ville de Panamá, les autres viennent du reste du pays. La moyenne des revenus est de $410 \$$ par mois, mais un tiers a moins de 300\$. L'instabilité des ressources et le bas niveau de qualification de la population enquêtée sont apparues très clairement comme des freins au maintien des familles présentes. Celle ci est rarement évoquée contrairement à ce qui se passe dans la plupart des centres historiques du continent

A cette situation sociale difficile s'ajoutent des conditions de logement inacceptables tant le processus de taudification et d'entassement est grave. 54,5\% des familles vivent en location et 24,5\% dans des maisons condamnées ${ }^{9}$, c'est- à-dire reconnues insalubres par le Ministère du logement et interdites à l'habitat.17, $4 \%$ en logements abandonnés. Ainsi, $42 \%$ des familles vivent en danger. Mais, bien que $55 \%$ des familles puissent payer un loyer modeste, il est impossible de trouver une solution sur place étant donné le type de restauration mis en place et en l'absence de toute volonté en faveur d'une composition sociale mixte et d'essai d'intervention comme celle du Pactarim ${ }^{10} .{ }^{11}$

De nouvelles interventions pour accélérer la récupération du centre historique

La récupération de ce site se fait, à la fois, par valorisation et par réintégration dans l'organisation de l'espace métropolitain comme conséquences d'interventions récentes tant du secteur public que privé, nationales et internationales.

Deux interventions ont accéléré le processus de transformation du centre historique

- L'UNESCO en reconnaissant, en 1997, le Casco Antiguo de Panamá comme Patrimoine de l'Humanité a valorisé immédiatement cet ensemble architectural et urbain, au tracé colonial encore présent, témoin d'une histoire exceptionnelle dans les relations entre l'Espagne et les pays andins ${ }^{12}$.

- Le décret- loi 9 de 1997 qui offrent des avantages fiscaux et des possibilités de crédit à ceux qui acquièrent un édifice et commencent à le réhabiliter dans un délai de deux ans.

Brusquement, naît un marché immobilier spéculatif s'ouvrant à l'image de ce qui se passe dans les villes comme Cartagena, San Juan et Antigua. Le Plan Maestro de 1998, mis en place par la Comisión del Casco Antiguo, offre enfin l'outil de travail. Réalisé à partir d'études approfondies dont une enquête socio-économique, un inventaire des maisons, il crée entre autre un SIG et met en place en 2000 l'Oficina para la Restauración y Puesta en valor del Conjunto Monumental Histórico del Casco Antiguo de la Ciudad de Panamá (OCA). Cette dernière compte avec une direction technique

\footnotetext{
${ }^{7}$ La restauration des immeubles de la fin du XIX e siècle et du début du XXe siècle situés dans la partie coloniale prouve bien le rejet volontaire de cette architecture résidentielle située autour de San Felipe

${ }^{8}$ Les trois corregimientos du centre ancien représentent 55073 habitants en 2000 répartis dans 79 manzanas.

${ }^{9}$ Insalubrité et hors norme sur le plan des équipements sanitaires avec de grands risques d'effondrement et d'incendie.

10 1,5\% des familles souhaitent rester dans San Felipe et 35\% un logement social du MIVI dans le quartier voisin d'El Chorillo.
}

\footnotetext{
${ }^{12}$ La ville fortifiée date de 1673 à la suite de l'abandon de Panama la vieja, premier site sur le Pacifique situé à quelques $\mathrm{km}$ à l'Est.
} 
chargée d'appliquer les orientations du Plan Maestro ${ }^{13}$. Enfin, une commission est chargée des demandes de permis de construire et d'aménagement des édifices pour vérifier la conformité avec les contraintes historiques et architecturales.

L'intervention plus intensive du secteur public, tant de l'Etat que de la Municipalité a eu un effet moteur sur le secteur privé. Outre l'achat de quelques immeubles et la restauration de monuments dont certains emblématiques du lieu ou du pouvoir, d'énormes travaux ont permis de modeler une image positive et attractive autour du palais présidentiel, du Musée du canal interocéanique, du Ministère de l'Intérieur et de Justice, du Ministère des Affaires étrangères et du Théâtre national occupant des édifices historiques de la fin du siècle dernier. Quelques immeubles d'origine coloniale mais surtout de la fin du XIX e siècle ont été expropriés, restaurés et transférés à des usages administratifs (comme la casa de los monogrammas au Fondo de Inversion Social qui dépend du Ministère de la Présidence). La présence du palais présidentiel offre une sécurité exceptionnelle à ce nouvel environnement urbain avec une présence policière encadrant, jour et nuit, les ruelles de la partie privilégiée. Les travaux d'aménagement de l'espace public sont visibles : rues et trottoirs, câblages souterrains, aménagement des places et des parkings, et illumination non seulement des rues mais également la mise en lumière des édifices. La coopération internationale participe largement à ces travaux.

Préparées à la vente par les interdictions à habiter ${ }^{14}$ du MIVI, les maisons aux fenêtres bouchées de parpaings et sur le point de s'écrouler, sont achetées, réhabilitées, puis vendues (1000 à 1200 \$ le m2) ou louées par appartement. Quelques rez-de-chaussée sont transformés en commerces ou restaurants.. L'offre est encore limitée et chère, une maison en ruine qui n'arrivait pas à se vendre, il y a quelques années trouve preneur aujourd'hui à $50000 \$$. Seulement, une dizaine de personnes et deux ou trois sociétés immobilières ont, pour l'instant, parié sur le Casco Antiguo. Il fallait proposer un style de vie original et lancer une mode locale et récupérer les atouts du monde cosmopolite résidant à Panamá autour d'approche psychologique confortant le "couple branché " en quête d'autre mode de logement que l'appartement en tour ou la villa-jardin.

L'espace intervenu est encore très limité, mais il contribue à des changements visibles et décisifs. Peu à peu, en moins de 10 ans, la valeur des édifices a augmenté entre $40 \%$ et $100 \%$. 15\% des 940 maisons ou édifices recensés ${ }^{15}$ ont été restaurés. Beaucoup d'autres sont en cours de transformation. Entre 50 et $60 \%$ ont été vendus de seconde main, à travers des agences immobilières à des européens et à des Américains. Un appartement de $50 \mathrm{~m} 2$ coûte 50 à $80000 \$$ selon la situation. La valeur de cette dernière l'emporte toujours sur la qualité des services (eau, électricité, téléphone) qui demeure le point faible du quartier. Ici, l'usage du sol est précis valorisant ainsi telle ou telle rue et limite la densité. Ainsi l'extrême pointe de la Péninsule, calle Oeste, l'Avenida A et l'Avenida Central ainsi que la place de la Cathédrale et la Plaza Herrera sont les plus recherchées ${ }^{16}$. Une quinzaine de cafés, restaurants et boutiques animent, ça et là, les rues étroites. Mais, on ne trouve aucun équipement commercial, de loisirs, d'éducation, de santé en relation avec le statut social des nouveaux habitants. Ceux-ci doivent être conformes aux lieux : jeunes sans enfants, cadres ou artistes.. En revanche, habiter là confère un statut social.

Le coût de la restauration est élevé $500 \$ 1$ e $\mathrm{m} 2$ en raison des matériaux utilisés souvent importés de Colombie. Des normes imposent que les structures physiques des édifices ne s'éloignent pas de l'apparence coloniale. De bon marché, les maisons sont devenues, en quelques années, chères même si le prix du $\mathrm{m} 2$ reste inférieur à celui d'une maison dans un quartier résidentiel. Toutefois, les deux années 2002 et 2003 enregistrent une diminution du nombre de projets de restauration alors que se sont mis en place les grands projets de restauration comme celui de la Cathédrale ou du Théâtre

\footnotetext{
${ }^{13}$ Le directeur de l'OCA est l'architecte Vanessa Spadafora.

${ }^{14}$ Le projet de restauration du casco Antiguo affichait en 200311 caserones et 2 pensions condamnées. Cette même année, le Mivi relogeait en appartement dans El Marañon plus de 70 familles de San Felipe.

${ }^{15}$ Dont 134 appartiennent au gouvernement, 16 à l'église catholique, le reste au secteur privé.

${ }^{16}$ Le manque de stationnement est aussi un problème
} 
National ${ }^{17}$. Ainsi contrairement à ce qui s'était passé dans la décennie 90 où les investissements du secteur privé était limité ${ }^{18}$ par rapport à ceux du centre d'affaires( CFI), le début de la décennie 2000 enregistre un regain d'intérêt pour le Casco Antiguo.

Le décret ayant décidé que seul le quartier de San Felipe était intéressant l'a sauvé, mais, a condamné les autres. Le défaut des secteurs voisins de Santa Ana et de Chorillo: n'exister que depuis la fin du XIX et début XX siècle. Ils sont doublement condamnés car personne ne sait quoi faire de ces quartiers trop populaires et trop pauvres en attendant que l'incendie élimine un par un ces édifices de bois témoins de cette architecture propre aux Caraïbes et en Amérique centrale. Leurs paysages d'habitats délabrés constituent une barrière psychologiquement pesante. La dynamique générale ne les concerne pas et pire il n'existe aucun projet les concernant car les autorités ne semblent pas savoir comment intervenir sur ces immeubles taudifiés. Cette impression, somme toute, est relative quand on voit l'afflux récent de fonctionnaires, nouveaux résidents aisés et touristes, passer sans état d'âme, cette ceinture urbaine populaire pour gagner le Casco Antiguo. Le processus de gentryfication est bien là et, de toutes façons, il n'a jamais été question de l'éviter. La partie réhabilitée ressemble, de plus en plus, avec ses maisons peintes à un décor de théâtre dans lequel on se promène plus qu'on y habite. Derrière les balcons fleuris qui cachent la misère du logement, aperçoit les vrais résidents qui attendent un relogement ailleurs.

Le changement d'échelle de la ville favorise le centre ancien

Le changement du centre ancien vient surtout de son insertion dans la demande de la ville tout entière qui, plus grande et plus active, a besoin d'un centre-ville plus vaste englobant tous les éléments de la centralité propres à une métropole de plus d'un million d'habitants et au caractère résolument mondial de certaines de ces activités. Inclure un quartier ancien, qui est de plus "patrimoine mondial" est devenu important non seulement dans le marketing urbain mais aussi dans la vie de Panamá.

En effet, la croissance de la ville et l'insertion de l'ex ville américaine pose la question du changement d'échelle de la nouvelle aire métropolitaine qui intervient de plusieurs façons :

- Sur le plan spatial, le centre historique excentré jusque là, par rapport à la marche vers l'est de l'urbanisation et des activités tertiaires et de décision, se retrouve au cœur du nouvel espace réunifié englobant la zone du canal et toutes les croissances périphériques de l'Ouest, au-delà des zones d'opérations techniques et des zones forestières de protection du canal même. La position du centre a tout simplement changé avec cette forte expansion marquée par des taux records vers Arraiján ${ }^{19}$ et par une grande anarchie urbanistique. Cette situation ne ralentie pas pour autant l'extension des affaires vers l'Est et l'urbanisation au-delà de l'aéroport de Tocumen.

- Sur le plan des activités, la concentration des lieux de pouvoir, c'est-à-dire, la centralité, a donc évolué. Avec la croissance générale de la ville sur le plan démographique et fonctionnel, Panama a besoin d'un centre-ville plus vaste que celui qui correspond au centre d'affaires. D'autant plus que plusieurs activités administratives et d'encadrement, sont restées dans les quartiers des années 1930 ou ont pris récemment une place dans la zone du canal. Cette centralité en apparence éclatée ne l'est pas si l'on considère les distances. Ainsi d'Ancón et Balboa, hauts lieux du commandement américain, au Centre Financier International (en cours de prolongement vers l'Est avec le centre de conventions), se forme l'espace centre-ville dont a besoin la capitale, avec à l'intérieur de cet ensemble des secteurs très différents mais participant, par leurs équipements et leurs fonctions, à la centralité générale. En se situant entre les zones logistiques et portuaires et le Centre International des Affaires, il est un point de passage obligé pour accéder à certaines administrations (le palais présidentiel et les deux ministères, celui de l'Intérieur et de la Justice et celui des Affaires Etrangères). Le Casco Antiguo est un des éléments de ce grand centre-ville.

\footnotetext{
${ }^{17}$ Le Ministère du logement (Mivi) a enregistré 47 projets en 2001, 38 en 2002 et 11 au premier semestre de 2003.

${ }^{18}$ A. Uribe, Tareas $\mathrm{p} 48$

${ }^{19} 150000$ habitants en 2000 contre 25000 en 1970
} 
- Sur le plan touristique, le centre historique est un parcours incontournable pour les touristes qui viennent de la péninsule d'Amador où se trouve le terminal des croisières mais aussi pour les nombreux congressistes qui viennent de la zone hôtelière située près du Centre Financier ${ }^{20}$. Le parcours va devoir s'élargir encore avec la récente inscription de Panamá la Vieja par l'UNESCO en juillet 2003. Située à l'Est, entre l'aéroport de Tocumen et le centre des affaires international, ce site archéologique conserve des ruines des édifices civils et religieux de la première ville coloniale sur l'océan Pacifique.

- Sur le plan des liaisons avec le reste de la ville, les aménagements récents conformes au Plan de Area Metropolitana de Panamá de 1998 ont cherché à rompre l'isolement en rapprochant la ville américaine et la zone du canal du reste de l'aire métropolitaine. L'amélioration de la jonction entre Balboa et la ville en particulier El Chorillo et Santa Ana est, aujourd'hui, assurée par cette voie rapide qui traverse toute la ville en passant au sud du cerro Ancón. Elle se terminait brutalement dans la partie américaine dont la traversée était autorisée pour gagner le pont ${ }^{21}$.La construction du deuxième pont qui s'achèvera en 2004 dédoublera le trafic trop élevé aux heures d'entrée et de sortie du travail qui engorge les avenues traditionnelles du centre-ville avec les flux provenant de l'Ouest . rappelons que la ville dispose d'une sorte de périphérique (corridors) qui relie les différents quartiers à l'autoroute (inachevée et coûteuse) de Colon.

Une partie de cette recomposition urbaine est liée à la dynamique logistique et touristique qui marque les premières années 2000 et dont fait partie le centre historique. Aujourd'hui, Panama, est au cœur d'une révolution portuaire intégrant les ports et le canal dans la logistique mondiale. Les espaces portuaires hérités des américains sont en pleine modernisation soutenue par le secteur privé des grands opérateurs internationaux (en particulier asiatiques) en matière de transport maritime. L'augmentation de la capacité de transit du canal est le grand défi du début du XXI e siècle.

La mise en place d'une politique patrimoniale adaptée à la nouvelle vocation touristique

La politique touristique mise en place, depuis 1998, comme contrepartie à cette extrême dépendance logistique et à la fonction bancaire, bénéficie du Plan Maestro de Desarrollo Turistico de la Republica de Panamá avec, en particulier, de son Plan Desarrollo Turistico Patrimonial mais aussi d'une campagne publicitaire de 10 millions de \$, lancée en 2001, dans les Amériques du Nord et du Sud, et, en Europe. 50000 habitants Selon l'Organisation du Tourisme Mondiale, le Panama enregistre une croissance moyenne de 7 à $9 \%$ par an avec des retombées locales importantes (de janvier à septembre 2002, 386 millions \$). L'accueil des touristes trouve désormais à côté des établissements de grand luxe (4 hôtels de 5 étoiles à Panama, 1 à Colon), apparaissent des hôtels de catégorie inférieure susceptible d'offrir des gammes de prix. Des forfaits sont alors proposés incluant des visites dans le quartier financier et commercial, le petit tour au Casco Antiguo (il faut bien un peu de culture) et l'approche du canal en assistant au passage d'un navire dans l'écluse de Miraflores. En moins d'une journée, les sites emblématiques de la capitale sont parcourus et il ne reste plus qu'à partir une semaine dans les îles de Bocas del Toro, sites promus internationalement au détriment du reste du Panamá.

Les deux façades océaniques contribuent-elles aussi à l'essor du centre historique de Panamá et de la même façon, Colon sur l'Atlantique. En effet, l'essor des croisières de la décennie passée se poursuit et favorise des ports sur leur parcours. Un terminal d'accueil a été créé à Amador et à Colon (Terminal 2000). Le projet pour Amador a connu des chemins d'hésitation. Trois îlots militaires chargés de garder l'entrée du Canal et les navires en attente dans la baie, ont été reliés au continent par des remblais facilitant leur accès. Plusieurs projets résidentiels et de loisirs se sont succédés. Hôtels, restaurants, port de plaisance ont pris place en attendant le vaste projet du musée de la biodiversité confié à l'architecte international Gerhi confortant la position d'accueil sur des bases diversifiées que souhaite acquérir Panama pour dépasser sa vocation bancaire et commerciales.

\footnotetext{
${ }^{20}$ La Compagnie aérienne panaméenne Copan assure une liaison avec toutes les grandes villes du continent.

${ }^{21}$ Quelques grillages au pied du cerro d'Ancón rappellent la militarisation de la zone. On savait, il y a 20 ans, qu'il ne fallait pas s'écarter de cette voie sous peine d'être interpellé
} 
Il est aisé d'accueillir des touristes pour une journée, et même de les faire venir de Colón, par le nouveau train, achevé en 2001, après une heure de voyage dont une partie sur le lac Gatún, mais il faut aussi les distraire. Il est hors de question de proposer la plage tant la baie est polluée par le trafic des navires mais aussi par la ville qui y déverse ses déchets. En attendant les travaux d'assainissement et les usines de traitement des eaux prévues, on prévoit de repenser les itinéraires entre le centre ancien et le centre moderne en réconciliant la ville avec son bord de mer. Le rivage reste beau offrant au coucher du soleil une vue superbe sur la baie unissant d'un côté la presqu'île du centre ancien aux édifices de deux à trois étages et la presqu'île de Paitilla hérissée de tours miroitantes sous les rayons solaires. Mais la promenade, Avenue Balboa, se fait le long d'une autoroute dangereuse à traverser, et passe par quelques quartiers réputés pour leur insécurité.

La recomposition urbaine sur une base patrimoniale se poursuit avec le projet La Exposición où le Gouverneur de la Province de Panama tente de mobiliser (août 2003) tous les acteurs institutionnels pour améliorer ce quartier des années 1910-30 et qui a gardé de beaux éléments d'architecture sans compter son plan en damier riche de places aménagées. Lieu de passage entre le Centre financier et les quartiers commerciaux populaires de Santa Ana et la zone portuaire, il est traversé par quatre axes bruyants et pollués faisant oublier qu'il est resté un quartier administratif et résidentiel. C'est ce dernier aspect qui fait de lui un quartier plus aisé à améliorer que les quartiers populaires de Santa Ana et d'El Chorillo.

Reconnu comme espace unique au monde, l'ex ville américaine bénéficie d'une nouvelle stratégie mise en place en 1998 intégrant la nature, la culture et l'histoire du canal et du pays. La zone du canal est alors utilisée comme attraction. L'écluse de Miraflores s'est équipée pour recevoir les touristes. Ainsi, les Panaméens découvrent que leur canal intéresse les étrangers qui effectuent la traversée du pays par le canal lors d'une journée de croisière.

Le plan de cité jardin de la ville américaine avec son édifice de l'administration, sa maison de l'administrateur et la disposition des édifices administratifs d'El Prado constitue en soi un patrimoine que les Panaméens ont su garder. Dans quelques années seulement, il sera, peut être, possible de proposer une patrimonialisation de la zone urbaine du canal, en se basant sur la reconnaissance du concept de cité jardin tropicale à la fois maritime et militaire. Mais, les esprits ne sont pas encore prêts. Les derniers militaires américains sont partis le 31 décembre 1999 et la sacralisation n'est pas encore de rigueur en observant les nombreux chantiers en cours entre la zone portuaire et Clayton : un échangeur, un agrandissement du port avec destruction de bâtiments obsolètes, un immense centre commercial et un terminal routier. La transformation de l'aéroport d'Albrook est souvent évoquée. On avait même pensé recréer une cité administrative en créant toute une série d'édifices en ligne comme à El Prado. La vocation administrative du quartier est déjà forte. Les ministères de la Education, de l'Agriculture, de la Santé ont reçu des dizaines d'hectares et des bâtiments.

\section{Conclusion}

Le grand défi proposé par l'architecte E. Tejeira est-il en train d'être gagné ${ }^{22}$ : relier le Casco Antiguo à Balboa, le canal, le centre financier et même la forêt présente à moins de deux kilomètres de ces quartiers grâce aux mesures de protection? Toutes ces interventions en centre historique ne sont pas nouvelles mais elles se sont renforcées, après des années d'attente, par une série d'interventions à la fin de la décennie 90 mobilisant à la fois le secteur public et le secteur privé pour permettre le décollage d'opérations à une autre échelle que les précédentes. Le secteur public est, de ce fait, largement intervenu dans le champs normatif, dans le financement et la conservation du patrimoine et, enfin, dans la fonctionnalité urbaine provoquant un effet d'entraînement pour le secteur privé.

Encouragés, les investissements ont suivi le modèle spéculatif classique d'un marché international sans se soucier des autres quartiers centraux et périphériques qui ne font pas partie de cette nouvelle fonctionnalité. On est en train de construire un décor pour une catégorie sociale selon le modèle de la

\footnotetext{
${ }^{22}$ El Casco Antiguo de la ciudad de Panamá,Publicacion de cultura metropoliltana,Edicion Ciudad 525 pages 2000
} 
gentryfication observée souvent au début de la recomposition territoriale et pour les touristes auxquels on propose un parcours diversifié de certains secteurs urbains choisis. Ce nouveau centre-ville est vaste puisqu'il englobe le Casco Antiguo, le quartier des années 1920-1930, puis le quartier des tours des années 70 à nos jours et bien sur l'ex ville américaine. Devenu un espace de consommation, il est le cœur de la ville des affaires et du tourisme mais l'est-il pour le reste de la ville ? Comment les autres quartiers et leurs habitants s'articulent- ils à cet espace central ? Peut-être est-il trop tôt pour affirmer que ces nouvelles interventions signifient une meilleure intégration urbaine. 